'Departamento de

Endocrinología. Escuela de Medicina. Pontificia Universidad Católica de Chile. Santiago, Chile. ${ }^{2}$ Departamento de Pediatría. Escuela de Medicina. Pontificia Universidad Católica de Chile. Santiago, Chile.

Los autores no declaran conflictos de interés.

Recibido el 20 de agosto de 2017, aceptado el 30 de enero de 2018.

Correspondencia a: Carolina Peña V.

Departamento de Endocrinología Pontificia Universidad Católica de Chile.

Diagonal Paraguay 362, piso 4. Santiago, Chile. Capena3@uc.cl

\title{
Pseudohipoparatiroidismo de presentación tardía: reporte de dos casos
}

\section{CAROLINA PEÑA ${ }^{1}$, CONSTANZA PINOCHET ${ }^{2}$, PABLO FLORENZANO ${ }^{1}$, CAROLINA MENDOZA², CAROLINA GARFIAS ${ }^{2}$ MARCELA ARACENA ${ }^{2}$, CECILIA MELLADO ${ }^{2}$, GILBERTO GONZÁLEZ ${ }^{1}$ \\ Pseudohypoparathyroidism: report of two cases of late presentation}

Pseudohypoparathyroidism (PHP) is a group of rare genetic disorders that share organ targeted resistance to the action of parathyroid hormone (PTH) as a common feature. Biochemically, they may present with hypocalcemia, hyperphosphatemia and elevated PTH. Some forms present with a specific phenotype: short stature, round facies, short neck, obesity, brachydactyly and subcutaneous calcifications, called Albrigth's Hereditary Osteodystrophy (AHO). This spectrum of disorders are caused by several alterations in the gene coding for the alpha subunit of the G protein (GNAS): an ubiquitous signaling protein that mediates the action of numerous hormones such as PTH, TSH, gonadotropins, and ACTH, among others. According to their inheritance with maternal or paternal imprinting, they may manifest in a diversity of clinical forms. Although most commonly diagnosed during childhood, PHP may manifest clinically during adolescence or early adulthood. We report two late presenting cases of pseudohypoparathyroidism. A 21-year-old female with biochemical abnormalities characteristic of pseudohypoparathyroidism who was misdiagnosed as epilepsy and a 13-year-old boy with the classic AHO phenotype but without alterations in phospho-calcium metabolism, compatible with pseudopseudohypoparathyrodism.

(Rev Med Chile 2018; 146: 116-121)

Key words: Calcium Metabolism Disorders; Fibrous Dysplasia, Polyostic; Phosphorus Metabolism Disorders; Pseudohypoparathyroidism; Pseudopseudohypoparathyroidism.

\section{Introducción}

1 l término pseudohipoparatiroidismo (PHP) comprende un grupo de trastornos genéticos infrecuentes, que tienen en común la resistencia a la acción de la paratohormona (PTH) en el túbulo renal proximal, afectando allí la excreción de fosfato y la síntesis de calcitriol, metabolito más activo de la vitamina D. El PHP se manifiesta bioquímicamente por hipocalcemia, hiperfosfemia y PTH elevada sin evidencia de falla renal y/o hipomagnesemia ${ }^{1}$. Algunas variantes de esta patología pueden presentar un fenotipo espe- cífico caracterizado por talla baja, facies redonda, cuello corto, obesidad, braquidactilia, osificación heterotópica y discapacidad intelectual variable, conocido como osteodistrofia hereditaria de Albright $(\mathrm{OHA})^{2}$. En otros casos de PHP, se observa solo fenotipo de OHA, pero sin manifestaciones bioquímicas de resistencia a $\mathrm{PTH}$, tales pacientes se denominan como pseudopseudohipopatiroidismo $(\mathrm{PPHP})^{3}$. La prevalencia estimada del PHP es de 0,79 por 100.000 habitantes $^{2}$.

Este conjunto de patologías se producen por mutaciones del gen GNAS, el cual codifica para la subunidad alfa de la proteína G $(\mathrm{Gs} \alpha)$. La 
Gs $\alpha$ es una proteína ubicua de señalización de receptores acoplados a proteína $\mathrm{G}$ que media la acción de hormonas como PTH, TSH, gonadotropinas y GHRH entre otras. La unión de estas hormonas a su receptor lleva a la activación de la adenilciclasa con el consecuente aumento del $\mathrm{AMPc}^{4}$. Mutaciones del GNAS generan disminución de la expresión y/o función de la Gs $\alpha$ lo que se manifiesta clínicamente como OHA con o sin resistencia hormonal ${ }^{5}$.

En Chile, a nuestro mejor entender, se han publicado sólo 2 casos de $\mathrm{PHP}^{6,7}$, correspondiendo uno de ellos a la forma clásica de presentación en la infancia ${ }^{6}$.

Nuestro objetivo es comunicar dos casos de PHP de presentación tardía, de variedades no clásicas de la enfermedad, para ilustrar la importancia clínica de reconocer oportunamente esta patología y evitar complicaciones graves con un tratamiento adecuado.

\section{Caso clínico 1}

Mujer de 21 años, universitaria, con antecedentes de hipotiroidismo primario no autoinmune desde los 16 años en tratamiento con levotiroxina y epilepsia de reciente diagnóstico. Tiene historia de parestesias peribucales y en manos desde la infancia. Un año previo a la consulta comenzó con crisis de ausencia, que posteriormente evolucionaron a convulsiones tónico-clónicas generalizadas. Se manejó con Carbamazepina. Antecedentes familiares: padre con hipotiroidismo y enfermedad renal crónica, madre con obesidad.

Entre sus exámenes destacan tomografía axial computarizada de cerebro que muestra depósitos cálcicos supra e infratentoriales (Figura 1). Exámenes de laboratorio: calcemia: $5,4 \mathrm{mg} / \mathrm{dL}$, albúmina: $4,5 \mathrm{~g} / \mathrm{dL}$, fosfemia: $8,1 \mathrm{mg} / \mathrm{dL}$, creatinina plasmática: 0,64 mg/dL, FA: 154 U/L, PTH: 171 pg/ mL, 25OHD: $20,7 \mathrm{ng} / \mathrm{mL}$. Al examen físico: Talla: $1,51 \mathrm{~m}$ (talla objetivo genético $1,49 \mathrm{~m}$ ), peso: 67 $\mathrm{k}$, IMC $28,3 \mathrm{~km}^{2}$, facies redonda, Chvostek $(+)$, sin calcificaciones subcutáneas. Se planteó PHP y se solicitó radiografía de manos que descartó braquidactilia y ecografía renal que no mostró nefrocalcinosis. El estudio bioquímico en padres no evidenció hipocalcemia. Se inició tratamiento oral con calcio elemental 4,0 g/d más calcitriol 0,25 ug cada $12 \mathrm{~h}$. Actualmente calcemia y PTH normales (Tabla 1) Sin nuevas crisis convulsivas, carbamazepina en disminución gradual.

\section{Caso clínico 2}

Adolescente de sexo masculino, de 13 años 7 meses en control irregular por talla baja desde los 6 años, se descartó déficit de hormona de cre-

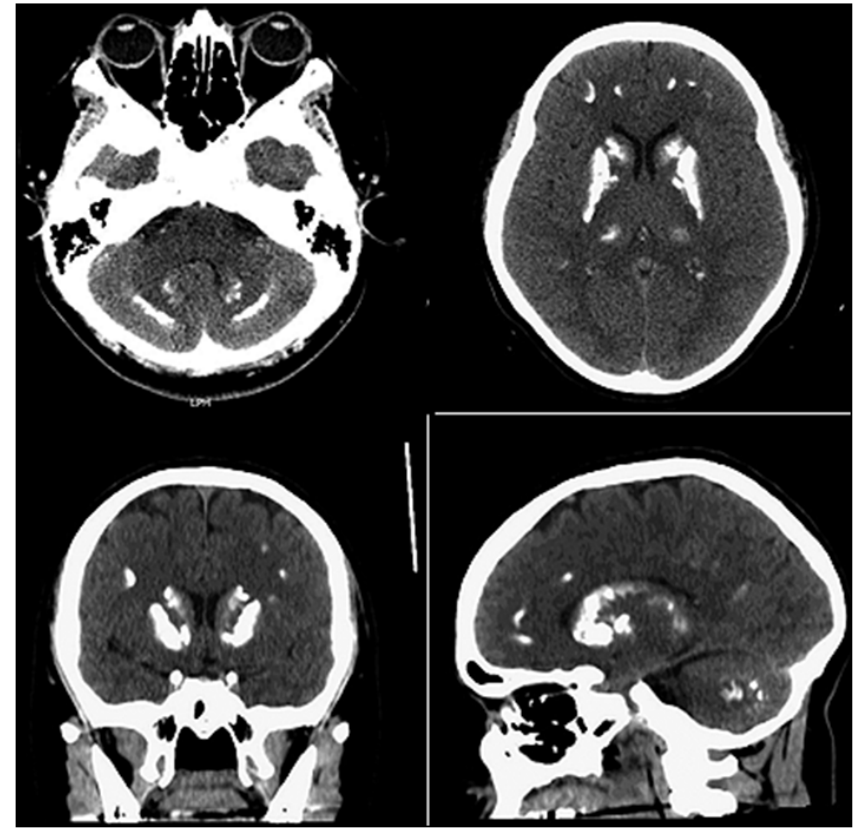

Figura 1. Caso clínico 1: Tomografía axial computarizada de cerebro. Calcificaciones en cerebelo, núcleos grises centrales, tálamo, sustancia blanca periventricular y subcortical. 
cimiento. Antecedente de aumento volumen en muslo derecho, el cual fue extirpado a los 10 años: biopsia compatible con paniculitis osificante. Sin antecedentes familiares relevantes.

Antecedentes perinatales: Recién nacido de término, pequeño para la edad gestacional. Desarrollo psicomotor normal, retraso leve en el área del lenguaje.

Examen físico: Talla de 1,42 m (-2,41 DS), peso: $34,3 \mathrm{~kg}$; IMC: $16,9 \mathrm{~km}^{2}$ (p 17\%). Enver- gadura: $138 \mathrm{~cm}$, relación segmento superior/ segmento inferior de 0,9 . Talla objetivo genético 1,74 m (p 36\%; + 0,33 DE). Cara redonda, paladar alto, pectum excavatum, vello púbico Tanner II, testes de $4 \mathrm{cc}$ a derecha y testículo izquierdo no palpable. Acortamiento de metacarpianos y metatarsianos.

Estudio radiológico confirma hallazgos (Figura 2). Exámenes de laboratorio sin alteraciones del metabolismo calcio fósforo (Tabla 1).

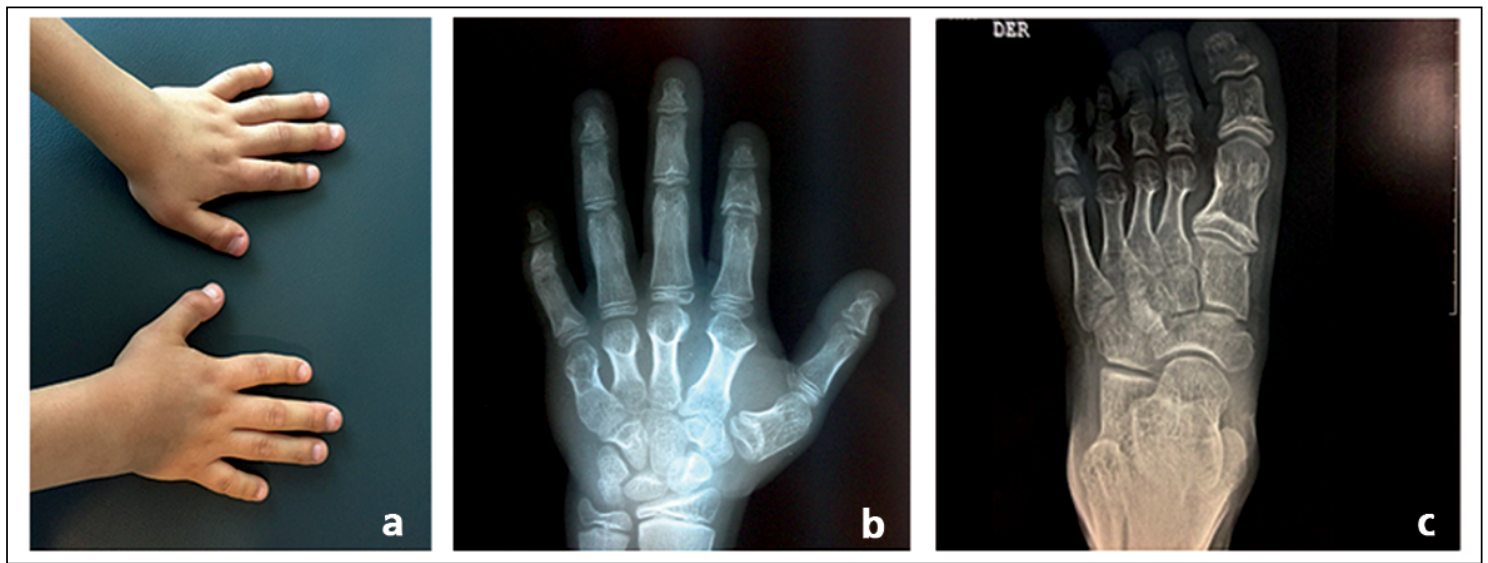

Figura 2. Caso clínico 2. Fotografía de manos. Radiografías de manos y pies. Se observa el acortamiento localizado de metacarpos tanto al examen físico como en el estudio radiológico (a) y (b), también se observó acortamiento de los metatarsianos en el estudio radiológico $(\mathbf{c})$.

Tabla 1. Exámenes caso clínico 1 y 2

\begin{tabular}{|c|c|c|c|c|c|}
\hline Examen & $\begin{array}{l}\text { Paciente } 1 \\
\text { Tiempo } 1\end{array}$ & $\begin{array}{l}\text { Paciente } 1 \\
\text { Tiempo } 2\end{array}$ & $\begin{array}{l}\text { Paciente } 2 \\
\text { Tiempo } 1\end{array}$ & $\begin{array}{c}\text { Paciente } 2 \\
\text { Tiempo } 2\end{array}$ & $\begin{array}{l}\text { Valor de } \\
\text { referencia }\end{array}$ \\
\hline $25(\mathrm{OH})$ Vitamina D (ng/dL) & 20 & - & 31,7 & & $>30$ \\
\hline PTH (pg/mL) & 171 & 66 & 67,7 & 31,6 & $15-65$ \\
\hline $\mathrm{Ca}(\mathrm{mg} / \mathrm{dL})$ & 5,4 & 9,5 & 9,7 & 10,3 & $8,5-10,5$ \\
\hline $\mathrm{Mg}(\mathrm{mg} / \mathrm{dL})$ & 2,0 & & 2,5 & & $1,6-2,5$ \\
\hline$P(m g / d L)$ & 7,4 & 5,1 & 4,1 & 4,5 & $2,6-4,5$ \\
\hline $\mathrm{FA}(\mathrm{U} / \mathrm{L})$ & 154 & - & 185 & 322 & $\begin{array}{l}\text { Niños: hasta } 350 \\
\text { Adultos: } 25 \text { - } 100\end{array}$ \\
\hline TSH (uUl/mL) & 4,59 & - & 3,92 & 2,91 & $0,3-4,2$ \\
\hline $\begin{array}{l}\text { T4 (ug/dL) } \\
\text { T4 libre (ng/dL) }\end{array}$ & 4,4 & - & 1,56 & 1,24 & $\begin{array}{l}4,6-12 \\
0,93-1,7\end{array}$ \\
\hline Ac TPO (UI/mL) & $<0,1$ & - & & & $<12$ \\
\hline Testosterona (ng/dL) & & $\mathrm{n} / \mathrm{c}$ & & 29 & $\begin{array}{l}13-17 \text { años } \\
28-1.110\end{array}$ \\
\hline $\mathrm{LH}(\mathrm{mUl} / \mathrm{mL})$ & & & & 1,93 & $1,5-9,3$ \\
\hline
\end{tabular}

Paciente 1: tiempo 1 corresponde a los exámenes tomados al momento del diagnóstico; tiempo 2: exámenes fueron tomados 2 meses luego del inicio del tratamiento con calcio y calcitrol. Paciente 2: tiempo 1: exámenes tomados durante la primera evaluación por endocrino pediátrico del paciente; tiempo 2: exámenes tomados durante la última evaluación del paciente 3 años posterior a los primeros, sin mediar intervención. Abreviaciones: PTH: paratohormona; Ca: Calcio; P: fósforo; FA: fosfatasas alcalinas; TSH: hormona tiroestimulante; Ac TPO: anticuerpos antiperoxidasa; LH: Hormona luteinizante; n/c: no corresponde. 


\section{Discusión}

La clasificación del PHP es compleja y ha evolucionado en relación a la mejor comprensión de los mecanismos moleculares implicados en su etiología. En la Tabla 2 se muestra la clasificación actual del PHP, basada en el defecto genético y fenotipo principal de cada variante. El PHP tipo Ia corresponde a la variedad más frecuente y clásica de PHP. A diferencia de éste, el PHP Ib carece de fenotipo OHA y además de la resistencia a PTH, sólo se ha descrito resistencia a TSH. El PHP Ia y Ic son clínicamente idénticos con presencia de fenotipo de OHA y resistencia hormonal múltiple. Sólo se diferencian en que el PHP Ia ha mostrado deficiencia parcial de la actividad de Gs $\alpha$ (alrededor 50\%) en membranas de otros tipos celulares (eritrocitos, fibroblastos y plaquetas), no así el PHP Ic. Como es de esperar secundario a la disminución de la actividad de la Gs $\alpha$ el PHP I no muestra aumento de los niveles de AMPc en orina luego de una infusión de PTH bovina. ${ }^{2} \mathrm{El}$ PPHP corresponde a una variante normocalcémica del PHP que carece de resistencias hormonales aunque los pacientes presentan $\mathrm{OHA}^{8}$.

Conocer las bases genéticas de esta patología representa un gran desafío, pero permite entender las manifestaciones clínicas características. El PHP se produce por mutaciones del GNAS o alteraciones en el imprinting de este gen. La transcripción de la Gs $\alpha$ es bialélica (expresión del alelo materno y paterno) en la mayoría de los tejidos, pero en algunos como túbulo renal proximal, tiroides, células somatótropas pituitarias y gónadas, se expresa sólo el alelo materno con silenciamiento del alelo paterno (mecanismo denominado imprinting). Los pacientes con PHP tipo 1a tienen mutaciones heterocigotas en los exones maternos del GNAS; mientras que los pacientes con PHP tipo $1 \mathrm{~b}$, tienen defectos de metilación en el locus GNAS que reducen la transcripción de la Gs $\alpha$ del alelo materno. Algunos pacientes pueden presentar también disomía uniparental, situación en que ambos alelos del GNAS son heredados del padre. Tanto en el PHP tipo la como $1 \mathrm{~b}$ el silenciamiento del alelo paterno produce clínicamente la resistencia a PTH y $\mathrm{TSH}^{1}$. Tabla 2. Por otra parte, el PPHP se produce cuando la mutación de GNAS es en el alelo paterno. Como mencionamos previamente, dado que GNAS se expresa de forma bialélica en la mayoría de los tejidos, (ej, hueso condrocitos y placa de crecimiento), se postula que en la OHA las características clínicas se producen por haploinsuficiencia (funcionamiento de solo un alelo) del GNAS en estos tejidos. La talla baja y la braquidactilia de la OHA pueden deberse a la fusión prematura de la epífisis en huesos largos y tubulares, lo que demuestra la necesidad de dos copias funcionales del GNAS para el crecimiento y maduración normal del cartílago de crecimiento ${ }^{5}$.

Tabla 2. Clasificación del Pseudohipoparatiroidismo. Modificado de ref $\mathbf{f}^{2,5,7}$

\begin{tabular}{|c|c|c|c|c|c|c|c|}
\hline Tipo & $\begin{array}{l}\text { Defecto } \\
\text { genético }\end{array}$ & $\begin{array}{c}\text { Origen } \\
\text { parental }\end{array}$ & $\begin{array}{c}\text { Fenotipo } \\
\text { OHA }\end{array}$ & $\begin{array}{c}\text { Resistencia } \\
\text { hormonal }\end{array}$ & $\mathrm{Ca} / \mathrm{P}$ & PTH & $\begin{array}{l}\text { AMPc Ur } \\
\text { post PTH }\end{array}$ \\
\hline la & $\begin{array}{l}\text { - Mutaciones heterocigotas } \\
\text { inactivantes ubicadas en los } \\
\text { exones 1-13 del gen GNAS }\end{array}$ & Materno & $\mathrm{Si}$ & $\begin{array}{l}\mathrm{PTH} / \mathrm{TSH} / \\
\text { FSH-LH/ } \\
\text { GHRH }\end{array}$ & $\downarrow \uparrow$ & $\uparrow$ & No \\
\hline $\mathrm{lb}$ & $\begin{array}{l}\text { - Mutaciones que afectan el } \\
\text { imprinting del GNAS tales } \\
\text { como microdeleciones en } \\
\text { STX16 o NESP55/AS del } \\
\text { gen GNAS) } \\
\text { - Disomía uniparental }\end{array}$ & Materno & No & $\begin{array}{l}\text { PTH } \\
\text { TSH }\end{array}$ & $\downarrow \uparrow$ & $\uparrow$ & No \\
\hline Ic & $\begin{array}{l}\text { - Mutaciones heterocigotas } \\
\text { inactivantes ubicadas en los } \\
\text { exones } 1-13 \text { del gen GNAS }\end{array}$ & Materno & Si & $\begin{array}{l}\mathrm{PTH} / \mathrm{TSH} / \\
\text { FSH-LH/ } \\
\text { GHRH }\end{array}$ & $\downarrow \uparrow$ & $\uparrow$ & No \\
\hline PPHP & - Mutaciones en GNAS & Paterno & $\mathrm{Si}$ & No & $\mathrm{N} / \mathrm{N}$ & $\mathrm{N}$ & Si \\
\hline
\end{tabular}

N: normal; OHA: Osteodistrofia hereditaria de Albright; AMPc Ur; AMP cíclico urinario; PPHP: Pseudopseudohipoparatiroidismo; Gsa: sub unidad Alpha de la proteína G. 
En el caso 1 se planteó el diagnóstico de PHP tipo Ib, dado hallazgos de laboratorio compatibles de PHP en ausencia de OHA. El hipotirodismo sin anticuerpos antitiroideos medibles se interpretó como resistencia a la TSH. Es posible que la ausencia de fenotipo de PHP explicara en parte el retraso en el diagnóstico de esta paciente, a pesar que presentaba síntomas sugerentes de hipocalcemia desde la infancia agravándose hasta convulsiones en la edad adulta que llevaron al diagnóstico errado de epilepsia. En un primer episodio convulsivo en pacientes adultos se describe que hasta un $15-30 \%$ son de causa tóxico- metabólicas. La hipocalcemia, principalmente aguda, puede estar presente en $20-30 \%$ de los casos de convulsiones en los adultos donde la presentanción como convulsión tónico-clónica es la más habitual ${ }^{9}$. Dado que no disponemos de calcemias previas, no es posible descartar que la hipocalcemia en este caso haya sido de aparición más tardía.

El segundo paciente correspondería a un PPHP ya que tiene elementos categóricos de OHA (talla baja, braquidactilia y osificación heterotópica), pero con normocalcemia ${ }^{10}$. Ambos casos, así, ilustran la presentación clínica variable incluso dentro de la heterogeneidad del PHP. El estudio molecular en búsqueda de mutaciones inactivantes del GNAS permite distinguir los distintos tipos de PHP. Sin embargo, ello no pudo realizarse en estos casos.

Respecto al tratamiento del PHP; éste se debe manejar con suplementación oral de calcio y calcitriol para lograr normocalcemia. Dado la resistencia de la acción de la PTH a nivel proximal el uso de colecalciferol o ergocalciferol no reporta beneficios en el manejo de las alteraciones bioquímicas. La nefrocalcinosis y la hipercalciuria son muy infrecuentes ya que la acción de la PTH a nivel distal del nefrón se encuentra conservada y por tanto la reabsorción tubular distal de calcio está preservada. Como no existe resistencia esquelética a la $\mathrm{PTH}$, la elevación sostenida de ésta en el PHP, puede asociarse a pérdida de masa ósea, por lo que el objetivo del tratamiento, junto con normalizar la calcemia, debe ser también reducir los niveles plasmáticos de PTH y evitar el sobretratamieno que puede generar hipercalcemia e hipercalciuria.

La resistencia a TSH y gonadotrofinas, que pueden acompañar al PHP, debe ser manejada de forma similar a cuando existe deficiencia de éstas causadas por otras etiologías ${ }^{11}$. Sin embargo, no existe evidencia categórica respecto a si pacientes con PHP y talla baja, deban ser tratados con hormona de crecimiento $(\mathrm{GH})$ ya que ésta es probablemente de origen multifactorial (cierre prematuro de los cartílagos de crecimiento, ausencia del estirón puberal, además de eventual déficit de GH). Se recomienda en tales casos una monitorización estricta de la talla y velocidad de crecimiento junto al desarrollo puberal ya que el reemplazo con $\mathrm{GH}$ estaría indicado para mejorar la talla baja durante el período de crecimiento lineal antes de la pubertad ${ }^{12}$. Dado la tendencia de estos pacientes a desarrollar obesidad se deben realizar intervenciones dietéticas y promover la actividad física. No hay terapia específica para las manifestaciones físicas y neurocognitivas de OHA. Las osificaciones heterotópicas no requieren cirugía a menos que causen dolor o deformidad ${ }^{11}$.

Se deben tener en cuenta complicaciones a largo plazo en el cuidado de pacientes con pseudohipoparatiroidismo, entre las que destacan:

- Cataratas y calcificaciones SNC: Secundarias a la elevacion del producto calcio-fósforo, presentes en PHP 1, pero no PHPP.

- Hiperparatiroidismo secundario / terciario: Se han descrito casos de Osteitis fibrosa quística, dado la que la actividad de PTH sobre el tejido óseo esta conservada. Además, la elevacion persitente de PTH puede inducir la aparición de hipercalcemia (hiperparatirodismo terciario), incluso requiriendo paratiroidectomía. Como ya se mencionó el riesgo de hipercalciuria / nefrocalcinosis inducida por el tratamiento con sales de calcio es bajo, dado la actividad persistente de PTH en el túbulo renal distal. Todo lo anterior hace recomendable buscar valores de PTH cercanos a lo normal ${ }^{13}$.

- Obesidad, Resistencia a la insulina y SAHOS La obesidad es un componente fenotipico central de la OHA. Esta se ha visto asociada a comorbilidades como SAHOS y resistencia a la insulina. Interesamente, se ha visto que la frecuencia de estas complicaiones es mayor en pacientes con PHP1a-c que en $\mathrm{PPHP}^{14,15}$.

Existen escasas publicaciones de casos de PHP en nuestro país. El primero de ellos descrito en 1986, correspondía a un niño de 8 años con fenotipo de OHA asociado a laboratorio compatible 
con resistencia a PTH (forma clásica de PHP tipo Ia) ${ }^{6}$. El segundo publicado el año 2004, un hombre de 28 años que se presentó con hipocalcemia y PTH muy alta sin fenotipo OHA, el que podría clasificarse como PHP tipo $1 \mathrm{~b}$ o PHP $2^{7}$.

En conclusión, frente a pacientes con síntomas o hallazgos de hipocalcemia, asociada a PTH elevada, con función renal normal, debe considerarse la posibilidad de PHP, incluso en etapa post puberal, ausencia de fenotipo OHA o de antecedentes familiares de tal patología. Es necesario descartar causas tóxico metabólicas en todos los adultos que debuten con episodios convulsivos.

El mejor conocimiento actual de la patogenia del PHP y la existencia creciente de técnicas de estudio molecular, permiten identificar mejor los distintos tipos de esta enfermedad,optimizar el manejo de sus complicaciones y realizar una adecuada consejería genética.

\section{Referencias}

1. Michael A. Levin. An uptodate on the clinical and molecular characteristics of pseudohypoparathyroidism. Curr Opin Endocrinol Diabetes Obes 2012; 19(6): 443-451.

2. Matovani G. Pseudohypoparathyroidism: Diagnosis and Treatment. J Clin Endocrinol Metab 2011; 96(10): 3020-30.

3. Bastepe M, Juppner H. GNAS Locus and Pseudohypoparathyrodism. Horm Res 2005; 63: 65-74.

4. Fernández-Rebolledo E, Lecumberri B, Gaztambide S, Martínez-Indart L, Pérez de Nanclares G, Castaño L, et al. Endocrine profile and phenotype-(Epi) genotype correlations in Spanish patients with pseudohypoparathyroidism. J Clin Endocrinol Metab 2013; 98 (5): E 996-E1006.
5. Turan S, Bastepe M. GNAS spectrum of disorders. Curr Osteoporos Rep 2015; 13: 146-58.

6. Abara S, Carranza C. Pseudohipoparatirodismo y cardiomiopatía hipocalcémica. Rev Chil Pediatr 1986; 57(6): 575-9.

7. López JM, Carrasco C. ¿Pseudohipoparatirodismo o déficit de vitamina D?. Rev Med Chile 2004; 132: 1527-31.

8. Primer on the metabolic bone diseases and disorders of mineral metabolism 5a edición, cap 44 pág 278-283.

9. Nardone R, Brigo F, Trinka E. Acute Symptomatic Seizures Caused by Electrolyte Disturbances. Journal of Clinical Neurology 2016; Jan 12(1): 21-33.

10. Simpson C, Grove E, Houston B.Pseudohypoparathyrodism. Lancet 2015; 385: 1123.

11. aMantovani G, Spada A, Elli F. Pseudohypoparathyroidism and $\mathrm{G}_{\mathrm{s}} \alpha$-cAMP-linked disorders: current view and open issues.Nature Reviews Endocrinology 2016; 12(6): 347-56.

12. Mantovani G, Ferrante E, Giavoli C. Recombinant human GH replacement therapy in children with pseudohypoparathyroidism type Ia: first study on the effect on growth. J Clin Endocrinol Metab 2010; 95: 5011-7.

13. Neary NM, El-Maouche D, Hopkins R. Development and treatment of tertiary hyperparathyrodism in patientes with pseudohypoparathyroidism type 1b. J Clin Endocrinol Metab 2012; Sep 97(9): 3025-30.

14. Long DN, McGuire S, Levine MA, Weinstein LS, Germain-Lee EL. Body mass index differences in pseudohypoparathyroidism type la versus pseudopseudohypoparathyroidism may implicate paternal imprinting of Galpha(s) in the development of human obesity. J Clin Endocrinol Metab 2007; Mar 92(3): 1073-9.

15. Muniyappa R, Warren MA, Zhao X Reduced insulin sensitivity in adults with pseudohypoparathyroidism type 1a. J Clin Endocrinol Metab 2013; Nov 98(11): E1796-801. 